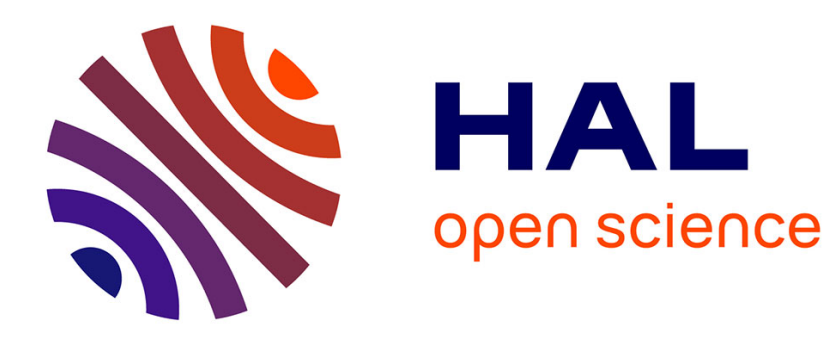

\title{
Quelle urbanisation au Levant Nord durant le 3e millénaire?
}

Christophe Nicolle

\section{To cite this version:}

Christophe Nicolle. Quelle urbanisation au Levant Nord durant le 3e millénaire?. Histoire urbaine, 2021, Cités millénaires du Proche-Orient, 61, pp.57-71. halshs-03529218

\section{HAL Id: halshs-03529218 \\ https://shs.hal.science/halshs-03529218}

Submitted on 17 Jan 2022

HAL is a multi-disciplinary open access archive for the deposit and dissemination of scientific research documents, whether they are published or not. The documents may come from teaching and research institutions in France or abroad, or from public or private research centers.
L'archive ouverte pluridisciplinaire HAL, est destinée au dépôt et à la diffusion de documents scientifiques de niveau recherche, publiés ou non, émanant des établissements d'enseignement et de recherche français ou étrangers, des laboratoires publics ou privés. 


\section{QUELLE URBANISATION AU LEVANT NORD DURANT LE IIIE MILLENAIRE?}

Christophe Nicolle (CNRS-Collège de France, PROCLAC)

La première urbanisation du Levant ${ }^{1}$ qui englobe les États modernes de Syrie, du Liban, d'Israël, de la Jordanie et de la Palestine se développe sur un temps long, de la fin du IV millénaire et durant le III $^{\mathrm{e}}$ millénaire avant notre ère ${ }^{2}$. Les sites fouillés attestent de l'émergence de nombreuses agglomérations dont certains sont interprétés comme des villes. De même que sa genèse est encore mal connue, les détails et les étapes de cette importante mutation nous échappent encore. Pourquoi les sociétés levantines qui vivaient jusqu'alors dans des agglomérations rurales, parfois de très grandes tailles ${ }^{3}$ inventèrent, à partir de la fin $\mathrm{du} \mathrm{IV}^{\mathrm{e}}$ millénaire, un nouveau type d'agglomération ? L'urbanisation levantine, comme d'autres urbanisations des périodes préclassiques, ne peut guère être résumée à un simple processus de concentration des populations dans les villes. Dans une société encore largement rurale et à dominante agricole, nul doute que cette mutation marquée par un « décollage organisationnel » avec un enrichissement de certains groupes sociaux et une capacité accrue de mobilisation des forces de travail n'a pu se faire qu'en s'appuyant sur l'élaboration d'un nouveau système agropastoral, d'un nouveau système agraire ${ }^{4}$. Mieux comprendre et décrire cette urbanisation passe donc tout autant par l'étude de ses morphologies et ses composantes urbaines que par la prise en compte de son nouveau rapport aux territoires.

L'urbanisation du Proche-Orient ancien a été un temps pensée comme un phénomène global, d'origine sud-mésopotamienne pour ensuite se diffuser aux régions alentour. Les différents mouvements d'urbanisation ont été expliqués en référence à ce «récit global» de

\footnotetext{
${ }^{1}$ Le Levant s'inscrit dans un carré virtuel de $650 \mathrm{~km}$ nord-sud sur $180 \mathrm{~km}$ est-ouest soit une superficie de $117000 \mathrm{~km}^{2}$. Une séparation traditionnellement est faite entre le Levant Nord et le Levant Sud. Bien qu'il s'agisse de concepts géoculturels dont la définition et les limites varient selon les périodes étudiées, pour les périodes protohistoriques et historiques, la séparation entre ces deux entités géographiques est placée à la latitude de Damas.

${ }^{2}$ Selon la périodisation en cours, du Bronze ancien II au Bronze ancien IV (env. 3150-2000 av. J.-C.).

${ }^{3}$ Certaines agglomérations des périodes préhistoriques et protohistoriques de la catégorie dite des «mégasites » atteignaient 40-90 ha.

${ }^{4}$ C'est-à-dire d'un mode d'exploitation du milieu adapté aux conditions environnementales et répondant aux besoins sociaux et économiques du moment. Cf. Hubert Cochet, "Origine et actualité du 'système agraire' : retour sur un concept », Revue Tiers Monde, n² 207/3, 2011, p. 97-114.
} 
l'urbanisation mésopotamienne et selon un schéma linéaire de l'histoire. L'avancée des recherches archéologiques a révélée et ne cesse d'apporter de nouvelles preuves de différences dans les morphologies urbaines proche-orientales signalant la concomitance des scénarios d'urbanisation et d'apparition des premiers États. Ces particularismes de rythme et de développement affirment l'importance des conditions locales qu'il s'agisse de celles des biotopes (dont la juxtaposition est une des caractéristiques du Levant) ou de la spécificité des organisations locales. Il n'y a donc pas une, mais des urbanisations du Proche-Orient que l'on décline en grandes aires culturelles (Mésopotamie du Sud et du Nord, Levant du Sud et du Nord, Anatolie, ...). Cependant, certaines similitudes formelles repérables dans plusieurs de ces scénarios locaux d'urbanisation signalent la possibilité de les envisager globalement, l'intérêt étant de permettre ainsi de pallier la discontinuité des données archéologiques disponibles.

\section{Une genèse urbaine incertaine et un développement en trois étapes}

Les plus anciennes attestations de l'urbanisation levantine proviennent de Palestine et de Jordanie. Elles datent du dernier quart du IV millénaire (entre 3250-2800 av. J.-C.). Dans un laps de temps qui n'excède pas 200 ans, une nouvelle catégorie de sites apparait et se diffuse. Ces établissements sont morphologiquement caractérisés par la présence des fortifications, des bâtiments publics et un habitat dense. La «fabrication» de ces nouveaux espaces construits reprend des éléments architecturaux (systèmes fortifiés ou aménagements hydrauliques $^{5}$ ) en usage dès le milieu du IV ${ }^{\mathrm{e}}$ millénaire. Elle n'en constitue pas moins une véritable rupture qualitative par rapport aux agglomérations de la période précédente. Les détails de cette transformation nous échappent encore. Plusieurs prospections archéologiques documentent pour cette période une forte diminution du nombre de sites occupés apparemment au profit de ces nouveaux sites fortifiés ${ }^{6}$. Il est possible que ces créations urbaines résultent d'une agrégation des habitants des villages alentour sans que l'on puisse établir s'il s'agit véritablement d'un synœcisme. Parmi les exemples relativement nombreux dans le sud du pays, nul cas ne vient documenter un processus graduel de transformation d'un village en ville. Il s'agit toujours soit de fondations ex nihilo, soit d'une transformation d'un site rural préexistant lors d'une réoccupation après une période d'abandon. Bien que le plus

\footnotetext{
${ }^{5}$ On trouve des exemples de ces aménagements par exemple sur le site de Jawa dans le Nord-est jordanien ou un peu plus tard sur celui de Khirbet el-Umbashi dans le sud de la Syrie ; Christophe Nicolle, «Entre symbiose et autarcie : les établissements fixes des pasteurs du Harra à l'âge du Bronze », Syria, n 88, 2011, p. 79-83.

${ }^{6}$ Pour une étude pionnière, voir : Magen Broshi et Ram Gophna, «The Settlements and Populations of Palestine during the Early Bronze Age II-III», Bulletin of the American Schools of Oriental Research, n 253, 1984, p. 4153.
} 
souvent très partielle, la fouille de quelques-uns de ces sites permet d'en dresser un portrait type. Dans une aire d'en moyenne 3-10 ha protégée par des fortifications dont certaines atteignent des dimensions monumentales, l'espace est occupé par un habitat urbain dense. Les maisons y sont agglutinées, organisées en ilots séparés par des rues, ou sur cour séparées les unes par rapport aux autres par des espaces résiduels servant aux circulations. Une autre caractéristique de ces agglomérations est l'omniprésence de bâtiments publics souvent en position topographique dominante. Il s'agit de temples, de palais et parfois de bâtiments aux fonctions plus incertaines : «maison de pouvoir », ou de lieux de réunion pour les élites locales. Dans la partie nord du Levant, les niveaux d'occupation de cette période n'ont été que très rarement dégagés. Un exemple de rempart connu dans le centre de la Syrie permet toutefois de supposer une certaine homogénéité de formes et de rythmes dans la genèse de cette première urbanisation levantine ${ }^{7}$.

Après cette période mal connue mais cruciale, on assiste à la propagation de cette nouvelle forme d'agglomération. La large diffusion de ce type d'agglomération à travers les différentes régions levantines atteste de son succès et de son adoption par différentes populations. La plaine côtière s'urbanise dès 2850/2800 av. J.-C. La célèbre Byblos passe du stade de village ouvert à celui d'une petite ville fortifiée d'au moins 5 ha en se dotant d'une enceinte sacrée et d'un temple. Elle est occupée par un réseau dense d'ilots réguliers de maisons ${ }^{8}$. D'autres indices de cette urbanisation côtière se retrouvent plus au sud à Sidon avec la découverte d'un entrepôt communal ou palatial. Il s'agit d'un grand bâtiment composé de huit pièces dont les capacités de stockage dépassent largement les besoins d'une famille ${ }^{9}$. Plus au nord, la petite ville côtière de Tell Fadous parait moins importante avec une superficie de seulement 1,5 ha. Mais avec son rempart, un escalier monumental d'accès et son habitat domestique dense séparé par des ruelles étroites, le site présente toutes les caractéristiques habituelles d'une agglomération urbaine. Plus à l'intérieur des terres, les indices se font rares. Dans l'ancienne

\footnotetext{
${ }^{7}$ Il s'agit du niveau II de Tell Afis avec une datation large entre 3800-3100 av. J.-C., cf. Stéphania Mazzoni, Serena Maria Cecchini, «Tell Afis (Siria) 1994, Rapporto Preliminare », Egitto e Vicino Oriente, n 18, 1995, p. 243-306.

${ }^{8}$ Jean Lauffray, Fouilles de Byblos, VI. L'urbanisme et l'architecture. Beyrouth, Bibliothèque Archéologique et Historique, $n^{\circ} 182,2008$, p. 67-187. Cette transformation se déroule durant la période dite du style « sableux», v. 3200-2700 av. J.-C. Le développement et l'enrichissement de la ville doivent beaucoup à son rôle dans les échanges maritimes principalement avec l'Égypte. Il faut envisager l'existence d'autres secteurs d'occupation aux alentours immédiats, notamment celle d'un port et de ses entrepôts. La superficie serait alors largement supérieure aux 5 ha actuellement connus, Francese Pinnock, «Byblos and Ebla in the 3rd Millennium BC. Two Urban Patterns in Comparison», ROSAPAT, $\mathrm{n}^{\circ}$ 4, 2007, p. 120, n. 15.

${ }^{9}$ Claude Doumet-Serhal, «Sidon during the Bronze Age: Burials, Rituals and Feasting Grounds at the "College Site"», Near Eastern Archaeology, n 73/2-3, 2010, p. 115.
} 
Ebla (Tell Mardikh) dont la superficie est alors évaluée à 7-8 ha $^{10}$, les fouilles ont partiellement dégagé les restes de ce qui est probablement un palais ${ }^{11}$. L'état général des fouilles en Syrie fait que cette découverte est un hapax. Malgré des données sporadiques, la comparaison avec le Sud mieux documenté permet d'envisager raisonnablement que, comme sur la côte, l'urbanisation se soit développée durant cette période en Syrie intérieure avec la mise en place de réseaux de villes fortifiées de 4-7 ha de superficie. Grâce à de récentes prospections, il est possible de noter des variations régionales de rythme et d'intensités dans les implantations selon l'attractivité des régions. C'est ainsi que la dépression endoréique du Matkh, une région de Syrie centrale apparait dès époque plus densément peuplée que d'autres régions alentour aux occupations plus clairsemées et plus tardives ${ }^{12}$. La proximité comme l'abondance des ressources en eau et la possibilité de pratiquer facilement une agriculture sèche apparaissent comme des facteurs déterminants dans l'intensification des occupations et le développement des premières villes.

Une troisième phase de l'urbanisation du Levant (2500-2000 av. J.-C.) correspond à une divergence radicale entre les scénarios du Nord et ceux du Sud. Au Sud, dès le début de la période, vers 2450/2500 av. J.-C., on assiste à un effondrement complet du système urbain qui structurait le pays depuis déjà près de 500 ans. Les villes de la période précédente, ainsi que nombreuses agglomérations rurales sont abandonnées. Les populations retournent à un mode de vie rural dans lequel le pastoralisme mobile joue un rôle important. Les raisons de cet effondrement et de cette transformation radicale du pays ne sont pas encore identifiées. Pour expliquer une telle disruption, des crises environnementales, socio-économiques sont

\footnotetext{
${ }^{10}$ Paolo Matthiae, «On this Side of the Euphrates. A Note on the Urban Origins in Inner Syria», dans Marcella. Frangipane, Helmut Hauptmann, Mario Liverani, Paolo Matthiae, Machteld Mellink (éditeurs.), Between the Rivers and over the Mountains. Archaeologica Anatolica et Mesopotamica Alba Palmieri Dedicata, Dipartimento di Scienze Storiche Archeologiche e Antropologiche dell'Antichità, Rome, Università di Roma «La Sapienza», 1993, p. 525.

11 Il s'agit principalement des bâtiments G2 et G5. Le bâtiment G2 dégagé sur une longueur de 30 m et une largeur de $10 \mathrm{~m}$, il comprenait au moins une dizaine de pièces. Selon son fouilleur il avait une fonction administrative. Du bâtiment G5, seules quatre pièces sont connues, mais avec des murs pouvant atteindre l'épaisseur conséquente de 1,50 m. Il n'est pas impossible que d'autres structures du Bronze ancien III aient été détruites lors de la construction de l'agglomération du Bronze ancien IVA. Paolo Matthiae, «Nouvelles fouilles à Ébla (1998-1999) : forts et palais de l'enceinte urbain», Comptes rendus de l'Académie des Inscriptions et belles Lettres, ${ }^{\circ}$ 144/2, 2000, p. 567-610.

${ }^{12} \mathrm{La}$ précocité de l'occupation de cette région d'environ $560 \mathrm{~km}^{2}$ s'explique par son attractivité due à une prédominance d'un environnement lacustre avec des terres bien arrosées et fertiles. Franscesca Baffi et Luca Peyronel, «Tell Tuqan and the Matkh Basin in a Regional Perspective. Thoughts and Questions Raised by the International Conference», dans Franscesca Baffi, Roberto Fiorentino et Luca Peyronel (éditeurs), Tell Tuqan Excavations and Regional Perspectives. Cultural Developments in inner Syria from the Early Bronze Age to the Persian/Hellenistic Period. Proceedings of the International Conference May 15th-17th 2013, Lecce, Galatina, Congedo Editore, 2014, p. 9-34.
} 
évoquées, des fins de cycle de croissance et de complexification sont proposées ${ }^{13}$. Elles ne constituent pas de réelles explications. Dans le même temps au Levant Nord, les attestations jusqu'alors discrètes de l'urbanisation se multiplient brusquement. Plusieurs agglomérations urbaines témoignent par leurs composantes et les artefacts découverts d'une acmé caractérisée tout autant par la multiplication des villes que par la richesse de leurs élites. Ce développement urbain s'intègre dans un mouvement de transformation de l'occupation du territoire dont témoigne l'amplification sans précédent du nombre et de la concentration des établissements urbains comme ruraux. Ainsi, dans le système fluviatile de la vallée de l'Oronte de la Syrie intérieure, les prospections attestent qu'il n'y a pas moins d'un site occupé tous les trois kilomètres ${ }^{14}$. Une rapide augmentation du nombre des occupations se constate dans d'autres régions comme la vallée du Matkh, la plaine du Jabboul ${ }^{15}$ où la plaine côtière. Là, sur une superficie de $450 \mathrm{~km}^{2}$ le territoire se couvre d'un maillage régulier de villages répartis autour de trois sites urbains couvre aussi le territoire ${ }^{16}$. Ce mouvement touche aussi des régions jusqu'alors inoccupées comme celle à l'est de l'Oronte ${ }^{17}$. Sur un réseau de wadis secondaires peu profonds jusqu'alors vides d'occupation se développe un réseau de 17 établissements probablement ruraux (env. 1-2 ha) implantés à intervalles réguliers sur deux wadis coulant de part et d'autre de la ville de Tell Mishrifé (25 ha $)^{18}$. D'autres régions, souvent de type endoréique, connaissent le même processus d'occupation comme la plaine du

\footnotetext{
${ }^{13}$ Kay Prag 2013, «The Southern Levant during the Intermediate Bronze Age», dans Margreet L. Steiner et Anne E. Killebrew (éditrices), The Oxford Handbook of the Archaeology of the Levant, c. 8000-332 BCE, Oxford, Oxford University Press, 2013, p. 397-398.

${ }^{14}$ Dans cette zone, la taille de ces sites varie entre 10-30 ha avec des sites comme Tell Ayu, Tell Afyun (moins de 20 ha) ou Tell an-Nasriya (30 ha), Karin Bartl et Michel al-Maqdissi, «Ancient Settlements in the Middle Orontes Region between ar-Rastan and Qal'at Shayzar. First results of Archaeological Surface Investigations 2003-2007», dans Daniele Morandi Bonacossi (éditeur), Urban and Natural Landscapes of an Ancient Syrian Capital. Settlement and Environment at Tell Mishrifeh/Qatna and in Central-Western Syria. Proceedings of the International Conference held in Udine, 9-11 December 2004, Udine, Forum Editrice Universitaria, Studi Archeologici su Qatna 1, 2007, p. 243-252.

${ }^{15}$ Pour la vallée du Matkh : Lucas Peyronel ,«Living Near the Lake. The Matkh Region (Syria) during the Early and Middle Bronze Ages», dans Francesca Baffi F., Roberto Fiorentino R. et Lucas Peyronel (éditeurs.), Tell Tuqan Excavations and Regional Perspectives. Cultural Developments in inner Syria from the Early Bronze Age to the Persian/Hellenistic Period. Proceedings of the International Conference May 15th-17th 2013, Lecce, Congedo Editore, 2014, p. 115-161; pour la plaine du Jabboul : Hans H. Curvers et Glenn Schwartz G., «Umm el-Marra, a Bronze Age Urban Center in the Jabbul Plain, Western Syria», American Journal of Archaeology, $n^{\circ}$ 101 (2), 1997, p. 201-227.

${ }^{16}$ Il s'agit de Tell Arqa, Tell Jamous et Tell Kazel ; Jean-Paul Thalmann, «Settlement Patterns and Agriculture in the Akkar Plain during the Late Early and Early Middle Bronze Ages», dans Daniele Morandi Bonacossi (éditeur), Urban and Natural Landscapes of an Ancient Syrian Capital. Settlement and Environment at Tell Mishrifeh/Qatna and in Central-Western Syria. Proceedings of the International Conference held in Udine, 9-11 December 2004, Udine, Forum Editrice Universitaria, Studi Archeologici su Qatna 1, 2007, p. 219-232.

${ }^{17}$ Graham Philip et al., «Settlement and landscape development in the Homs region, Syria: Report on work undertaken during 2001-2003», Levant, n³7, 2005, p. 21-42.

${ }^{18}$ Daniele Morandi Bonacossi, «Tell Mishrifeh and its Region during the EB IV and the EBA-MBA Transition. A First Assessment», dans Peter J. Parr (éditeur), The Levant in Transition. Proceedings of a Conference Held at the British Museum on 20-21 April 2004, Leeds, Palestinian Exploration Fund Annual Series, n 9, 2009 , p. 61.
} 
Jaboul avec pas moins de 47 sites occupés autour du principal centre urbain d'Umm el-Marra qui atteint alors une superficie de $27 \mathrm{ha}^{19}$. Ce mouvement expansionniste touche jusqu'aux zones les plus inhospitalières à la sédentarité du fait d'un déficit des ressources hydriques. Les marges orientales semi-arides de l'est jusqu'alors occupées épisodiquement par des pasteurs nomades sont profondément transformées par une importante vague migratoire venue de l'ouest et profitant d'une période d'optimum climatique. Une culture urbaine originale s'y développe avec la fondation de plusieurs villes rondes fortifiées aux superficies variant de 10 à $25 \mathrm{ha}^{20}$. La fondation de ces villes s'accompagne de la création de nombreux sites temporaires installés dans les wadis majeurs ou autour de faydas ${ }^{21}$. Sur ces nouvelles terres agricoles, population urbaine sédentaire et population mobile sont en étroites interconnexions.

Si la plupart des villes du Levant Nord sont de taille modeste (le plus souvent entre 5-15 ha), plusieurs d'entre elles se distinguent par leur composition et leur taille, atteignant des superficies de 25 à 50 ha. Elles appartiennent à une catégorie de capitales d'importance suprarégionale que l'on peut considérer comme des cités-États. Ébla, dont la superficie est alors estimée à 55 ha est l'une d'entre elles. Elle est connue par son palais royal (Palais G) dégagé sur $2500 \mathrm{~m}^{2}$ dont la superficie totale est estimée à $10000 \mathrm{~m}^{2}$. Il s'organisait autour d'une cour d'audience de $42 \mathrm{~m}$ de côté sur laquelle ouvrait une entrée monumentale large de $2,70 \mathrm{~m}$ qui se poursuivait par un escalier de marches en basalte menant aux secteurs supérieurs du bâtiment royal étagé dans la pente du tell. Près de 17000 tablettes cunéiformes découvertes dans une des pièces du palais documentent principalement les trois dernières années du royaume ${ }^{22}$. Par ailleurs, de nombreuses bullae et de nombreux objets attestent de la richesse de la famille royale qui occupait l'endroit ${ }^{23}$. Les autres bâtiments connus de la ville sont : au sommet du tell, un sanctuaire dynastique (le temple rouge) et en ville basse dans le

\footnotetext{
${ }^{19}$ Hans Curvers et Glenn Schwartz, «Umm el-Marra, a Bronze Age Urban Center in the Jabbul Plain, Western Syria», American Journal of Archaeology n 101/2, 1997, p. 201-227.

${ }^{20}$ Quatre exemples de ces villes sont connus : Tell el-Rawda (10,55 ha), Tell Khirbet Al-Qasr (11,27 ha), Tell Sh'airat (25 ha) et Tell al-Şūr (9,5 ha).

${ }^{21}$ Bernard Geyer et Yves Calvet, «Les steppes arides de la Syrie du Nord au Bronze ancien ou "la première conquête de l'est"'), dans Bernard Geyer (éditeur), Conquête de la steppe et appropriation des terres sur les marges arides $d u$ Croissant fertile, Lyon, Maison de l'Orient Méditerranéen-Jean Pouilloux, Travaux de la Maison de l'Orient, $\mathrm{n}^{\circ}$ 36, p. 55-88.

${ }^{22}$ Paolo Matthiae, «Ebla-Tell Mardikh, une capitale de la Syrie intérieure», Archéo-Théma, n 15, 2011, p. 3439.

${ }^{23}$ Avec par exemple la découverte dans la pièce L.9583 d'un étendard cérémoniel orné des statuettes en or et argent des reines Dusigu et Tabur-Damu et dans la pièce L.2866 d'un grand nombre de fragments (kaunakès, jupes, chevelures, etc.) de petites statues composites avec des revêtements en or et des incrustations de lapislazuli. Autre témoignage de la richesse du palais, en 2003, on arrivait à $17 \mathrm{~kg}$ de lapis-lazuli découvert dans cette seule pièce L.2866. Paolo Matthiae, «Temples et reines de l'Ebla protosyrienne. Résultats des fouilles à Tell Mardikh en 2007 et 2008», Comptes rendus des séances de l'Académie des Inscriptions et Belles-Lettres, $\mathrm{n}^{\circ} 153-2,2009$, p. 746-792.
} 
secteur oriental, un temple à antes monumental (le temple au rocher) mesurant 24,20 m sur 17 $\mathrm{m}$ avec des murs d'une épaisseur de $6 \mathrm{~m}^{24}$. À l'inverse du Sud, l'urbanisation dans le Nord ne connait pas de rupture ni même de déclin, hormis des destructions ponctuelles de villes résultant d'affrontements entre cités-États ${ }^{25}$, d'un retrait des régions semi-désertiques de l'est du fait de la fin de l'optimum climatique et des évolutions inhérentes à des changements culturels. Au contraire, la seconde moitié du $\mathrm{III}^{\mathrm{e}}$ millénaire semble avoir été une période de prospérité et de stabilité relative jusque vers 2000 av. J.-C. Ici, l'urbanisation a réussi son développement en satisfaisant l'ensemble de ses populations.

\section{Du formel à l'informel, la fabrique des villes levantines}

Au Levant comme dans le reste du Proche-Orient, les archéologues déterminent l'existence de villes avec la présence de plusieurs composants architecturaux considérés, pour des raisons, purement théoriques, caractéristiques d'un établissement urbain et d'un niveau d'organisation sociale. Identifier une ville peut paraitre de prime abord un exercice relativement simple, surtout si l'on se réfère aux schémas habituellement utilisés encore largement influencés par la théorie holistique de «Révolution urbaine » proposée en 1950 par Vere Gordon Childe ${ }^{26}$. Paradoxalement, malgré le titre de son article, son modèle ne s'attarde pas tant à décrire ce qu'est l'urbanisation que d'envisager toute la série de changements sociaux, économiques, politiques et culturels qui conduisent aux premiers États. La large adoption de sa théorie par les archéologues, notamment dans le domaine de l'archéologie du Levant Sud, s'explique surtout parce qu'il ne s'agissait pas d'un modèle purement spéculatif. Il s'appuyait sur des données archéologiques pour étayer l'argumentation de son processus ${ }^{27}$. Ce succès s'explique aussi dans sa formulation de dix critères simples permettant de distinguer les premières villes des villages contemporains ou plus anciens ${ }^{28}$. Des critères, aisément identifiables et donc faciles à utiliser par les archéologues orientalistes. Parmi eux, la présence de palais et de grands bâtiments comme celle d'entrepôts et de fortifications signalant selon son inventeur

\footnotetext{
24 Selon l'hypothèse du fouilleur Paolo Matthiae, il s'agirait du temple de Kura mentionnée dans le« rituel de la Royauté » des archives éblaites à proximité de la «porte de Kura », une des portes de la ville. Paolo Matthiae, «Temples and Queens at Ebla. Recent Discoveries in a Syrian Metropolis between Mesopotamia, Egypt and Levant», dans Interconnections in the Eastern Mediterranean. Lebanon in the Bronze and Iron Ages. Proceedings of the International Symposium, Beirut 2008, BAAL Hors Série, n VI, 2009, p. 117-139.

${ }^{25}$ Notamment la destruction d'Ébla par sa rivale Mari, cf. Alfonso Archi et Maria Giovanna Biga, «A Victory over Mari and the Fall of Ebla », Journal of Cuneiform Studies, $n^{\circ} 55,2003$, p. 1-44.

${ }^{26}$ Vere Gordon Childe, «The Urban Revolution», The Town Planning Review $\mathrm{n}^{\circ} 21,1950$, p. 3-17.

27 À l'époque il s'agissait des fouilles alors récentes d'Uruk.

28 Sur l'influence encore très forte en archéologie et dans d'autres sciences humaines de l'approche childienne : Michael E. Smith, «V. Gordon Childe and the Urban Revolution: An Historical Perspective on a Revolution in Urban Studies», Town Planning Review, n 80,2009 , p. 3-29.
} 
l'existence d'une société socialement stratifiée dotée d'un gouvernement centralisé. Il pratiquait une économie de centralisation-redistribution générant un surplus agricole qui permettait de dégager une partie de la population des activités agricoles. Pour autant, la caractérisation du stade urbain d'une agglomération à partir de ses seules composantes architecturales est encore une tâche ardue. Par exemple, la présence d'un système fortifié est jugée révélatrice d'une autonomie politique et d'un pouvoir coercitif capable de mobiliser une partie de la population pour sa construction. Le fait que des fortifications apparaissent dès le milieu du $\mathrm{IV}^{\mathrm{e}}$ millénaire sur certains sites à l'évidence non urbains et que la force de travail nécessaire à leur construction ne soit pas nécessairement importante indiquent que leurs présences sur un site ne permettent pas de préjuger de la nature urbaine ni de l'existence d'une certaine forme d'organisation sociale ${ }^{29}$. Les palais constituent une autre catégorie importante d'éléments architecturaux dans l'identification d'une ville et la mise en évidence d'un pouvoir hiérarchique et centralisé. Le Levant Sud se caractérise par la variété du faible nombre de palais. Dans ces palais comme partout ailleurs dans le pays, nulle trace d'écriture ou d'outils de gestion habituellement associés au développement d'une administration palatiale. Cette caractéristique et la variété des plans des quelques palais et des bâtiments que l'on pourrait ranger dans la catégorie de «bâtiments de pouvoir » signalent la coexistence de plusieurs formes de pouvoir de nature souvent plus hétérarchique que hiérarchisée. Au Levant Nord, il faut attendre la seconde moitié du III $^{\mathrm{e}}$ millénaire pour disposer d'un exemple de palais royal avéré, celui d'Ebla. Les archives du palais documentent une intense activité économique, des traités d'alliance passés avec d'autres Cités-États de l'est. Il est intéressant de noter que par plusieurs aspects, la royauté éblaite s'est inspirée de quelques pratiques et traditions mésopotamiennes en les adaptant à la situation locale ${ }^{30}$, comme s'il n'y avait pas eu au préalable de traditions royales équivalentes dans la zone levantine. Dans le domaine du religieux, les temples de cette période de plan in antis témoignent pour leur part d'une

\footnotetext{
${ }^{29}$ Fortifier un site d'une superficie moyenne de 10 ha (soit une population théorique de 2500 personnes) par une enceinte de $3 \mathrm{~m}$ de large et d'une hauteur de $10 \mathrm{~m}$ demande de construire une muraille de $1200 \mathrm{~m}$ de longueur et d'un volume moyen de $36000 \mathrm{~m}^{3}$ de blocs de pierre ou de briques crues. Si l'on considère que $0,9 \mathrm{~m}^{3}$ de briques peut être mis en place par jour de travail, une telle muraille pouvait être achevée en 40000 jours travaillés. Selon ces estimations (Raphaël Greenberg et al. «The Circles Building (Granary) at Tel Bet Yerah (Khirbet el-Kerak): A New Synthesis (Excavations of 1945-1946, 2003-2015)», Bulletin of the American Schools of Oriental Research, $n^{\circ} 378,2017$, p. 196) si environ 31-47 \% de la population était capable de se consacrer à ce travail durant les périodes creuses d'un calendrier agricole en hiver, deux à trois mois étaient alors suffisant pour achever une telle construction.

${ }^{30}$ Amalia Catagnoti, «Ebla », dans Raymond Westbrook (dir.), A History of Ancient Near Eastern Law, vol. 1, Leyde, Brill, coll. « Handbuch der Orientalistik », 2003, p. 227-239.
} 
certaine communauté de pensée et d'organisation dans la sphère levantine du religieux ${ }^{31}$. La seule approche typologique des agglomérations urbaines apparait donc peu efficace pour évaluer la nature des organisations socio-économiques qui peuvent y être associées. L'homogénéité de ces composantes urbaines ne dissimule pas pour autant la polysémie des différents établissements urbains. Il est évident que la ville d'Ebla avec son grand palais royal, ses temples et sa superficie de 55 ha n'avait pas grand-chose à voir avec la petite ville côtière de 1,5 ha de Tell Fadous. La diversité caractérise d'ailleurs les villes levantines non seulement dans leur morphologie et leur niveau de développement, mais aussi dans leur fonction. Le centre de certaines villes était consacré à des activités artisanales ou à des activités de stockage. Au cœur de la ville de Qatna, se sont succédé sur plusieurs niveaux des éléments de stockage destinés au traitement de céréales ${ }^{32}$. Dans une autre ville, celle de Tell Tuqan, on note la présence de silos similaires (Tuqan IC2 phases 6-7) remplacés par la suite par des installations et des fours de potier (phases 9-10) ${ }^{33}$. Ces villes ne sont pas seulement des outils économiques, elles sont parfois le lieu d'une mise en scène à une légitimation politique. À Ebla, les textes révèlent que le temple au rocher construit près de la «porte de Kura » faisait partie d'une déambulation scénarisée destinée à promouvoir les souverains de la ville dans leur rôle d'intercesseur royal avec les divinités. ${ }^{34}$ Une mise en scène d'un autre ordre est connue plus au nord-est dans la ville d'Umm al-Mara. Une nécropole particulière occupait le centre de la ville. Elle réunissait dans un complexe délimité par un mur d'enceinte une dizaine de tombes contenait un riche matériel funéraire composé d'objets en or, argent et de lapislazuli. Ces tombes étaient associées à des sacrifices d'équidés. La position des tombes au centre de la ville et la richesse du matériel funéraire proclament sans ambiguïté le haut rang des personnages qui y furent inhumés. À l'évidence, il s'agissait d'une mise en scène d'ancêtres défunts, des individus d'une même lignée ou d'une même famille régnante. Mais ici, hasard des fouilles ou réalité de la situation, pas de trace de palais royal. Quelle que soit la

\footnotetext{
${ }^{31}$ Pour un rappel des différents exemples de temples in antis connus au Levant au Bronze ancien de Meggido à Ebla : Corinne Castel, «The First Temples in antis: The Sanctuary of Tell Al-Rawda in the Context of 3rd millennium Syria», dans Jörg Becker, Ralph Hempelmann, Ellen Rehm (éditeurs), Kulturlandschaft Syrien, Zentrum und Peripherie, Festschrift für Jan-Waalke Meyer, Alte Orient und Altes Testament, $\mathrm{n}^{\circ} 371,2010$, p. 128.

32 Un grand grenier (Phases J38-37), des silos phases J36-J19) et des aménagements (des bassins phase J 36) : Daniele Morandi Bonacossi, «Excavations on the Acropolis of Mishrifeh, Operation J. A New Early Bronze Age III-Iron Age III. Sequence for Central Inner Syria. Part 1: Stratigraphy, Chronology and Architecture», Akkadica, $\mathrm{n}^{\circ}$ 128/1, 2008, p. 55-127.

${ }^{33}$ Luca Peyronel, «The Lower Town. Area P », dans Franscesca Baffi, Roberto Fiorentino et Luca Peyronel (éditeurs), Tell Tuqan Excavations and Regional Perspectives, op. cit., p. 9-34.

34 Paolo Matthiae, «Temples and Queens at Ebla. Recent Discoveries in a Syrian Metropolis between Mesopotamia, Egypt and Levant», dans Interconnections in the Eastern Mediterranean. Lebanon in the Bronze and Iron Ages, op. cit.
} 
nature des pouvoirs, hiérarchisés ou hétérarchiques, leur monopolisation par une association topographique et visuelle du centre des villes souligne la place prépondérante qu'elles y avaient. L'occupation par des monuments leur sont dédiés et des scénographies urbaines apparaissent comme le moyen aux nouvelles élites urbaines de légitimer et d'affirmer leur prééminence dans les villes et sur leurs habitants.

\section{Urbanisation et nouvelles stratégies agropastorales}

Le développement de l'urbanisation du Levant Nord durant la seconde moitié du III millénaire est concomitant d'un mouvement d'expansion territoriale. Il se caractérise tout autant par une concentration des maillages régionaux d'occupation que par la conquête et l'exploitation de nouveaux territoires agricoles, avec un éloignement des zones les plus anciennement occupées, car proches des ressources en eau. Les nouvelles zones conquises se trouvent pour l'essentiel à l'est d'une « ligne des puits » qui sépare la zone occidentale où la nappe phréatique est relativement proche de la surface et peut être captée par des puits et la zone orientale où les nappes ne sont plus accessibles ou tout simplement absentes. Il faut alors remplacer les puits par des citernes et l'agriculture irriguée extensive n'est plus envisageable $\mathrm{e}^{35}$. Cette dichotomie des ressources hydriques fait que plusieurs chercheurs considèrent qu'une telle expansion territoriale n'a été possible que grâce à certaines innovations ou adoptions technologiques (tel que l'invention des citernes, le développement de systèmes hydrauliques ou encore l'adoption de l'araire à soc de métal couplée à celle d'attelage d'ânes ou de bœufs). Deux constats contredisent ce postulat technologique. D’abord, les premiers systèmes hydrauliques clairement associés à des agglomérations sont connus dès le milieu du $\mathrm{IV}^{\mathrm{e}}$ millénaire ${ }^{36}$. Ensuite, aucun exemplaire d'araire à soc de métal n'a été retrouvé au Levant ${ }^{37}$. Par contre, des exemples d'outils aratoires en basalte ou en calcaire ont été découverts sur plusieurs sites des marges arides de l'est ${ }^{38}$. Des expérimentations faites avec ces outils ont montré qu'ils convenaient parfaitement à la culture

\footnotetext{
${ }^{35}$ Pour cette mention d'une « ligne des puits », Antoine Poidebard, La trace de Rome dans le désert de Syrie, Bibliothèque Archéologique et Historique $\mathrm{n}^{\circ}$ XVIII, Paris, 1934, p. 22-23.

${ }^{36}$ Notamment à Jawa en Jordanie du Nord et Khirbet Umbashi en Syrie du Sud, Frank Braemer et al., «Conquest of new lands and water systems in the western Fertile Crescent (Central and Southern Syria)», Water History, $\mathrm{n}^{\circ}$ 2, 2010, p. 102-104.

37 L'usage de l'araire en bois, dont l'invention remonte possiblement aux $\mathrm{VIII}^{\mathrm{e}}-\mathrm{VII}^{\mathrm{e}}$ millénaires est iconographiquement attesté au IV ${ }^{\mathrm{e}}$ millénaire en Mésopotamie. Andrew Sherratt, «Plough and Pastoralism: Aspects of the Secondary Products Revolution », dans Ian Hodder, Norman Hammond, Glynn Isaac (éditeurs), Pattern of the Past: Studies in Honour of David Clarke, Cambridge, Academic Press, 1981, p. 261-305.

${ }^{38}$ Notamment à Hama, Qatna, Tell Masin ; cf. Corinne Castel, « Des outils agricoles en pierre du Bronze ancien de Syrie », dans Bérangère Perello et Aline Tenu (éditrices), Parcours d'Orient. Mélanges offerts à Christine Kepinski, Oxford, 2016, p. 49-60.
} 
de terres encroutées semblables à celles des plateaux à l'est de l'Oronte ${ }^{39}$. Ainsi, l'hypothèse $\mathrm{du}$ franchissement d'un seuil technique comme facteur permettant le mouvement expansionniste nordiste ne tient pas. Les techniques employées n'expliquent pas les causes de cette «conquête » orientale, elles ne permettent que d'en souligner les modalités. Cependant, même si on ne sait pas encore l'expliquer, il est évident que ce mouvement d'expansion territoriale participe et a permis une amplification du développement et de l'enrichissement des populations urbaines septentrionales et de leurs élites.

Dans le Nord comme dans le Sud dans la première moitié du III $^{\mathrm{e}}$ millénaire, les nombreuses découvertes archéologiques dans les centres urbains, de greniers, d'entrepôts ou de salles contenant des jarres de stockage signalent l'importance de la production agricole et de son organisation dans les stratégies d'enrichissement de développement des pouvoirs et de développement des cultures urbaines notamment par le biais de l'essor d'une économie de centralisation et de redistribution ${ }^{40}$. On y trouve aussi les premières attestations d'une «économie palatiale urbaine » émergente ${ }^{41}$. Dans une seconde étape, celle de son apogée urbain, une importante mutation est perceptible dans le Nord avec l'émergence d'une économie développée non plus seulement sur la production agricole, mais aussi sur une production artisanale de masse (poterie, tissu, etc.), la production et l'échange de matériaux et de produits divers rares (or, lapis lazuli, bronze, etc.) dont on retrouve de nombreuses attestations à Ebla ${ }^{42}$. Dans le Sud, les élites n'ont pas atteint atteints ce stade d'organisation avant de disparaître dans l'effondrement du système urbain vers 2500 av. J.-C. Pour expliquer

\footnotetext{
${ }^{39}$ Axel Steensberg, Stone Shares of Ploughing Implements from the Bronze Age of Syria; A contribution to the Early History of the Ard-Plough, Copenhague, Historisk-filosofiske meddeleser Danske videnkabernes selskab, $\mathrm{n}^{\circ} 47,1977$. La découverte d'outils en pierre dans les marges arides confirme la proposition de cet auteur de la bonne adaptation de ces outils à la nature des sols steppiques car ils sont permettent de tracer des sillons suffisamment profonds pour retenir l'eau et permettre aux plantes de croitre; donc nulle besoin de faire appel à des araires en métal.

${ }^{40}$ La documentation disponible provient essentiellement du Levant Sud. On y relève en particulier que le matériel funéraire dans les tombes des élites se rapporte à une richesse agricole. Les objets manufacturés ou les matériaux rares sont généralement absents. Mérédith Chesson et Graham Philip, «Tales of the City? 'Urbanism' in the Early Bronze Age Levant from Mediterranean and Levantine Perspectives», Journal of Mediterranean Archaeology, $\mathrm{n}^{\circ}$ 16/1, 2003, p. 9-10.

$41 \mathrm{Au}$ Nord, les bâtiments G2 et G5 d'Ebla déjà mentionnés et dans le Sud principalement l'exemple du palais découvert à Tel Yarmouth. Pierre de Miroschedji, «Early Bronze Age Palaces in the Southern Levant», dans Manfred Bietak, Paolo Matthiae et Silvia Prell (éditeurs), Ancient Egyptian and Ancient Near Eastern Palaces, Volume II. Proceedings of a workshop held at the 10th ICAANE in Vienna, 25-26 April 2016, Harrassowitz Verlag, Wiesbaden, 2019, p. 159-179.

42 Cette modélisation du «Staple Finance » a été développée par des anthropologues américains pour expliquer la méthode d'enrichissement des petits états agraires mis en opposition avec celle de la «Wealth Finance » qui permet de dégager du profit à partir de productions artisanales de masse et de commerce avec des conversions possibles en produits agricoles. Dans le cas de sociétés à dominante agricole, même celles devenues, il y a une interpénétration entre ces deux modes de production. Terence N. D’Altroy et Timothy K. Earle, «Staple Finance, Wealth Finance, and Storage in the Inka Political Economy », Current Anthropology, n 26/2, 1985, p. 187-188.
} 
l'absence d'une transition similaire du «Staple finance » au Wealth finance » et du décollage organisationnel qu'il permet, les raisons invocables sont multiples : incapacité à développer des outils de gestion permettant d'amplifier la production agricole, le niveau de stockage et les échanges à plus longue distance, éloignement par rapport au monde mésopotamien, des régions agricoles moins riches et de plus petites dimensions, etc. La raison est probablement d'un autre ordre et concerner la nature de la structure sociale de ces élites.

Dans une société sans écriture, on ne connait pas grand-chose de ces organisations. Il convient toutefois de rappeler la place importante tenue par la cellule familiale dans les sociétés préclassiques. La maisonnée y apparait souvent comme une unité de base pour toutes formes d'organisation sociale dont. ${ }^{43}$. Il est alors envisageable que l'amplification à partir de 2500 av. J.-C. de l'urbanisation du Nord participe et accompagne la croissance et de l'institutionnalisation de certaines élites. Elles purent ou surent s'extraire du cadre restreint de la maisonnée familiale «naturelle » pour se projeter et se reconstruire dans un nouveau cadre institutionnel en développant une nouvelle forme d'économie palatiale dont l'échelle et la transversalité nécessitaient le recours à de nouveaux outils de gestion et de contrôle comme l'écriture et la pratique du scellement. La formation d'une aristocratie foncière accompagne le troisième stade de croissance du mouvement d'urbanisation du Levant Nord. La nouvelle richesse de cette aristocratie naissance s'appuie sur une économie de bien qui s'appuie encore pour une part importante sur la production agricole. Grâce à son pouvoir coercitif, ses capacités organisationnelles et d'investissements, elle joue sans nul doute un rôle moteur dans le mouvement d'expansion agricole. Les villes apparaissent comme l'outil de ce développement et de mises en valeur de nouveaux territoires agricoles. A la fois centres de centralisation et d'échange, de stockage, de contrôle territorial et lieux de légitimation des élites et de la manifestation de leur puissance.

\section{Logique urbaine et logique d'occupation des sols}

De toute évidence, il existe une corrélation entre le développement urbain et le changement du mode de production agricole qui caractérise l'urbanisation du Levant Nord dans la seconde moitié du III $^{\mathrm{e}}$ millénaire. Il est possible de théoriser les rythmes en s'inspirant des travaux des géographes Pierre Birot et Jean Dresch sur les mouvements d'expansion d'occupation à partir

\footnotetext{
${ }^{43} \mathrm{Du} \mathrm{III}^{\mathrm{e}}$ au I $\mathrm{e}^{\mathrm{e}}$ millénaire av. N.-É., il y a des attestations témoignant de l'importance de la maisonnée comme structure des sociétés proches-orientales ; voir principalement David J. Schloen, The House of the Father as Fact and Symbol. Patrimonialism in Ugarit and the Ancient Near East, Leiden, Studies in the Archaeology and History of the Levant, $\mathrm{n}^{\circ} 2,2001$.
} 
des zones de polyculture méditerranéenne classique ${ }^{44}$ vers la plaine alluviale et repérée sur la côte du Levant Nord par le fouilleur de Tell Arqa ${ }^{45}$. Une première phase de cette urbanisation serait marquée par le développement de grandes communautés agricoles installées dans les régions les plus propices (fin du Chalcolithique et début du Bronze ancien). Une seconde phase verrait, dans les mêmes zones, la transformation de ces communautés avec l'apparition et la multiplication des sites urbains et le développement d'élite s'appuyant sur la pratique et le contrôle d'une économie de productions agricoles (Staple Finance). Enfin, une troisième phase correspondrait à l'effondrement $\mathrm{du}$ système au Sud incapable d'opérer sa transformation structurelle et atteignant ses limites de fonctionnement. Les élites du Nord, sans doute déjà structurellement différentes, auraient été, elles capables de se réinventer, de se transformer grâce et conjointement à la mise en place d'une économie de bien (Wealth Finance) et au développement de systèmes de gestion plus efficaces. La conquête et l'exploitation de nouveaux territoires agricoles participent à cet enrichissement. La ville apparait comme l'endroit idoine par la construction de bâtiments (palais, tombes royales, temples dynastiques) et leur mise en valeur symbolique dans une mise en scène de ces nouvelles élites pour leur légitimation et de la pérennisation de leur pouvoir devenu héréditaire. L'urbanisation levantine peut donc se comprendre comme un mouvement de créations de villes, de développement et d'enrichissement d'une aristocratie foncière et d'augmentation du niveau de production agricole et artisanale. Des différences structurelles entre le Nord et le Sud signalent qu'il ne s'agisse pas de mouvements convergents. Urbanisation et développement agricole qui apparaissent dans de nombreux cas contradictoires sont dans le cas présent intégrés dans un même processus. La ville levantine du III $^{\mathrm{e}}$ millénaire n'est pas destructrice des espaces cultivés, mais elle est un outil dans le développement d'un nouveau système agraire.

\footnotetext{
${ }^{44}$ En se fondant sur des régularités induites par des similitudes du milieu, ces géographes ont proposés des scénarios récurrents d'occupation pour les plaines côtière de la Méditerranée orientale ; Pierre Birot et Jean Dresch, La Méditerranée et le Moyen Orient (2e éd), La Méditerranée orientale et le Moyen-Orient, les Balkans, l'Asie Mineure, Le Moyen-Orient, Paris, Presses Universitaires de France.

${ }^{45}$ Jean-Paul Thalmann, Tell Arqa - I. Les niveaux de l'âge du Bronze, Bibliothèque Archéologique et Historique, $\mathrm{n}^{\circ}$ 177, Beyrouth, 2006, p. 209-218.
} 